Campos Neutrais - Revista Latino-Americana de Relações Internacionais Vol. 1, No 3, p. 56-69, Setembro- Dezembro de 2019

\title{
Estudos de caso sobre formação de agenda no Brasil
}

\author{
Damasio Duval Rodrigues Neto* \\ Márcio Barcelos** \\ Rodrigo Serpa Pinto***
}

Resumo: Este é um artigo teórico, de cunho exploratório, no qual apresenta-se revisões das principais teorias da literatura sobre formação de agenda e de estudos que as aplicam em casos concretos no Brasil. O objetivo é realizar uma aproximação da literatura sobre formação de agenda com o ambiente da formulação de políticas públicas no país. Busca-se promover mais conhecimento sobre a utilização dos modelos revisados no contexto brasileiro e identificar pontos de congruência, visando assim suprir a lacuna referente à utilização destes modelos no contexto brasileiro. Conclui-se que conceitos das abordagens em questão, como imagem de política, janela de oportunidade e empreendedor de políticas podem ser utilizados para analisar a formulação de políticas no Brasil. A principal limitação deste artigo é a base de dados limitada, pelo qual não se pretende apresentar resultados taxativos. Novos estudos poderão trazer contribuições ao revisar outros casos e outras teorias sobre formação de agenda.

Palavras-chave: Formação de agenda. Políticas Públicas. Formulação de políticas.

\section{Agenda setting case studies in Brazil}

\begin{abstract}
This exploratory study presents a review of two of the most notable theories about agenda setting and of other studies that apply them in case studies in Brasil. The goal is to begin to approximate the agenda setting literature with the policy making environment in this country. This goal also aims to promote more knowledge about the application of the theories reviewed in this environment and identify converging points, as such looking to supply the lack of studies about the application of these theories in Brasil. The main limitation of this study is its small sample size, for which it does not seek to present conclusive results. New studies will bring contributions by reviewing other case studies and others agenda setting theories.

\footnotetext{
* Mestrado em Administração Pública pela Universidade Federal de Pelotas. Assistente em Administração na Universidade Federal de Pelotas.

** Professor do Departamento de Administração da Faculdade de Administração e Turismo da Universidade Federal de Pelotas (FAT-UFPel) e do Mestrado Profissional em Administração Pública (PROFIAP).

*** Professor Adjunto da Universidade Federal de Pelotas (UFPel) e Coordenador Local do Mestrado Profissional em Administração Pública em Rede Nacional (PROFIAP).
} 
Campos Neutrais - Revista Latino-Americana de Relações Internacionais Vol. 1, No 3, p. 56-69, Setembro- Dezembro de 2019

Keywords: Agenda setting. Public Policy. Policy making.

\section{Introdução}

A literatura sobre formação de agenda tem sido desenvolvida desde os anos 1970 (ZAHARIADIS, 2016) e seus principais modelos teóricos foram baseados no contexto norte americano. Kingdon (2014), em seu modelo dos "Múltiplos Fluxos", apresentado em 1984, analisa as áreas da saúde e transporte público, e Baumgartner e Jones (1993), na "Teoria do Equilíbrio Pontuado", analisam padrões de mudança em políticas públicas em diversos subsistemas, ambos nos Estados Unidos da América.

Estes dois modelos, tão destacados em diversas revisões teóricas e trabalhos empíricos (BRASIL, 2017; JONES e CAIRNEY, 2016; ZAHARIADIS, 2016; BARCELOS, 2015; CAPELLA, 2006), serão objeto de revisão, neste estudo, e os resultados de sua aplicação empírica em alguns estudos realizados no Brasil (RODRIGUES NETO, 2018; BRASIL, 2017; BARCELOS, 2015; METTEN et al, 2015; ZAPELINI, 2014), brevemente comentados. Trata-se de um estudo exploratório, com o objetivo de realizar uma aproximação da literatura sobre formação de agenda com o ambiente da formulação de políticas públicas no Brasil. Não se pretende esgotar a discussão ou apresentar resultados taxativos. Deu-se preferência a estudos recentes que tenham utilizados os modelos de Kingdon (2014) e Baumgartner e Jones (1993) diretamente em estudos de casos no Brasil. Especialmente, pela profundidade das investigações, as teses e dissertações revisadas (RODRIGUES NETO, 2018; BRASIL, 2017; BARCELOS, 2015) ofereceram os principais subsídios para a análise, com os artigos, mais concisos (METTEN et al, 2015; ZAPELINI, 2014), oferecendo recursos residuais.

Busca-se, portanto, promover a discussão sobre a utilização de duas das mais destacadas abordagens teórico-analíticas sobre formação de agenda e identificar os principais pontos de congruência e eventuais limitações de uma aplicação direta para o contexto brasileiro. De fato, Brasil (2017), em comentário sobre o uso da "Teoria do Equilíbrio Pontuado" como base de análise, afirma que foi uma escolha extremamente complexa de ser operacionalizada, por não ter encontrado, "na literatura nacional, trabalhos anteriores que aplicassem o Equilíbrio Pontuado como base principal de análise de políticas no Brasil" (BRASIL, 2017, p. 250). Da mesma forma, Zapelini (2014) afirma que a "Teoria dos Múltiplos Fluxos" "foi pouco aplicado empiricamente fora dos Estados Unidos" (ZAPELINI, 2014, p. 797). Foi exatamente com a intenção de contribuir para suprir esta lacuna nos estudos sobre políticas públicas no Brasil, que se realizou esta revisão. 
Assim, o artigo divide-se em três seções além desta introdução e das considerações finais. A seção a seguir apresenta, de maneira sintética, uma visão panorâmica da Teoria dos Múltiplos Fluxos, com sua ênfase no papel das ideias e na construção de problemas públicos. Após, na seção seguinte, é feita uma apresentação, também em forma condensada e concisa, dos aspectos essenciais que estruturam a Teoria do Equilíbrio Pontuado, com sua ênfase em subsistemas de política pública (policy subsystems), monopólios e mecanismos de feedback. Finalmente, na terceira seção discute-se a maneira como essas abordagens foram utilizadas em três estudos empíricos recentes que buscaram analisar processos de formação de agenda no contexto brasileiro.

\section{A Teoria dos Múltiplos Fluxos}

Em um dos modelos mais influentes na literatura sobre formação de agenda, Kingdon (2014) apresenta a "Teoria dos Múltiplos Fluxos", um modelo fluido, não linear e, a sua época, inovador. Desde então, praticamente todos os estudos sobre formação de agenda dialogam, de uma forma ou outra, com Kingdon (ROCHEFORT, 2016).

Desta abordagem, provém o conceito de agenda utilizado neste estudo - "a lista de assuntos ou problemas aos quais os governantes e pessoas de fora do governo associadas a eles estão prestando séria atenção em um determinado momento" (KINGDON, 2014, p. 3).

A grande novidade do modelo de Kingdon (2014) foi romper com a lógica dos estágios do "ciclo de política", predominante até os anos 1980. A partir de sua pesquisa, o autor conclui que a formulação de políticas públicas é muito menos rígida e racional do que entendia-se na ciência política tradicional. Suas conclusões remetem ao modelo da "lata de lixo", de Cohen, March e Olsen (1972) e a conceitos universais como ambiguidade, competição por atenção e racionalidade limitada (JONES e CAIRNEY, 2016; KINGDON, 2014).

$\mathrm{Na}$ “Teoria dos Múltiplos Fluxos", nega-se a lógica da formulação de políticas públicas como solução de problemas. De acordo com Kingdon (2014), as propostas de políticas públicas são formuladas por especialistas e pessoas que têm conhecimento técnico sobre o tema em questão e desenvolvidas ao longo do tempo por atores que investem recursos pessoais naquela proposta, com a intenção de auferir um ganho futuro - estes são os “empreendedores de políticas”. Devido à complexidade do sistema governamental, não seria possível que estes empreendedores e formuladores de políticas em geral esperassem a materialização das demandas para aí sim desenvolver as propostas. O que ocorre, afirma o autor, é que estes atores formulam suas propostas e as desenvolvem por um longo tempo, 
tentando "encaixá-las" aos problemas que aparecerem. Problemas e soluções, portanto, são fluxos relativamente independentes, que seguem de acordo com sua lógica própria. O outro fluxo é o da política, referente à política processual. A conversão dos fluxos dá oportunidade à mudança na agenda (KINGDON, 2014).

A noção de relativa independência entre os fluxos e a sua convergência motivada pela ação dos atores participantes, através de ideias e narrativas, faz do modelo de Kingdon (2014) um componente importante da literatura que analisa o papel das ideias nos processos de formulação de políticas. Em suma, Kingdon (2014) investiga como ideias são transformadas em soluções para problemas de política (JONES e CAIRNEY, 2016).

O entendimento de problemas, neste modelo, é de que são as questões de política reconhecidas pelas autoridades como problemas passíveis de serem objeto de uma tomada de decisão. O governo e as autoridades públicas em geral não têm condições de atender a todas as demandas sociais, portanto acabam escolhendo umas e ignorando outras. Aquelas que são escolhidas constituem os problemas reconhecidos.

Soluções são as propostas de política, formuladas pelos especialistas e desenvolvidas durante muito tempo, discutidas e recombinadas entre os atores da comunidade específica e eventualmente apresentadas ao grande público como "balões de ensaio". Estas propostas são propelidas em grande parte pela ação dos "empreendedores de política" e os atores envolvidos normalmente têm de esperar e persistir, aguardando uma oportunidade e tentando encaixar sua solução aos problemas que ascenderem à agenda. É o fluxo mais técnico e o que menos influi diretamente sobre a agenda, pois as propostas somente chegam nela quando problemas ou demandas políticas criam oportunidades para tal.

Já o fluxo da política diz respeito à dimensão processual do sistema, baseada nas negociações políticas, influenciadas principalmente pelo que Kingdon chama de "humor nacional", pela ação dos grupos de interesse e pelas mudanças dentro do próprio governo. $\mathrm{O}$ autor destaca a importância da figura do presidente da república na definição da agenda, afirmando que nenhum outro ator tem tanta capacidade de definir agendas. Não se trata de um poder absoluto, pois eventos fora do seu controle podem sobrepôr-se, mas, especialmente quando comparado a outros atores singulares, o presidente possui recursos extraordinários para influenciar na formação da agenda (KINGDON, 2014; CAPELLA, 2006).

Sob determinadas circunstâncias, os três fluxos convergem e assim configura-se o que Kingdon (2014) chama de "janelas de oportunidade”. São momentos em que há um problema reconhecido, as condições políticas são favoráveis e há uma solução disponível. Estas circunstâncias são desencadeadas pelo fluxo dos problemas ou da política e os 
empreendedores apresentam suas propostas como soluções para as demandas desses fluxos. Após apresentar suas alternativas de política e desencadear os eventos da janela de oportunidade, os participantes perdem o controle sobre o processo e os resultados tornam-se imprevisíveis. Emendas e até novas questões podem ser inseridas e novos atores podem entrar no processo. Assim, os outputs de políticas públicas estão sujeitos a um processo imprevisível e muito menos lógico do que propunha a ciência política tradicional (KINGDON, 2014; CAPELLA, 2006).

A "Teoria dos Múltiplos Fluxos" introduziu a análise do papel das ideias, da atuação dos empreendedores, da ambiguidade, racionalidade limitada e imprevisibilidade na formulação de política. Os principais modelos teóricos desenvolvidos deste então utilizam muito do que foi proposto por nesta abordagem. Entre eles, talvez o mais amplamente utilizado e discutido, a "Teoria do Equilíbrio Pontuado", traz novos olhares e direcionamentos diferentes, porém a influência de Kingdon se faz presente, na consideração das ideias, na importância dos empreendedores de política, na disputa pela atenção das autoridades e na conexão entre problemas e soluções, entre outras contribuições.

\section{A Teoria do Equilíbrio Pontuado}

A abordagem de Baumgartner e Jones (1993) pode ser melhor entendida a partir da noção de subsistema de política. Subsistema é uma comunidade específica, formada por especialistas em um determinado assunto, que tratam das questões de política referentes. Os subsistemas são comumente dominados por um monopólio de política, formado por atores que compartilham uma mesma ideia e apresentam uma mesma imagem de política sobre as questões a serem tratadas na comunidade. Além do entendimento conceitual comum, os monopólios também desenvolvem um arranjo institucional favorável, através do qual estabelecem espaços onde a tomada de decisão costuma estar de acordo com o entendimento vigente sobre as questões de política do subsistema (GREEN-PEDERSEN e PRINCEN, 2016; BAUMGARTNER e JONES, 1993).

Assim, dificilmente novas ideias e novas imagens conseguem ser introduzidas e por isto os subsistemas de política tendem à inércia. Propostas que desafiem o status quo daquele ambiente são rechaçadas, seu desenvolvimento é dificultado pela estrutura estabelecida e a tomada de decisão tende a favorecer o monopólio. Neste contexto, observa-se longos períodos de estabilidade.

Porém, Baumgartner e Jones (1993) carregam muito da base teórica de E. E. Schattschneider em sua abordagem, especialmente no que diz respeito à noção de expansão de 
conflito (GREEN-PEDERSEN e PRINCEN, 2016). O estabelecimento do monopólio de política coloca determinados atores em uma posição privilegiada no subsistema, os quais têm maiores possibilidades de promover suas ideias e apresentar propostas de política bem sucedidas. Enquanto os conflitos sobre as questões de política de interesse daquele subsistema estiverem restritos aos especialistas, a tendência é que o monopólio de política exerça sua força e suprima ideias contrárias à imagem de política preponderante, ao que Baumgartner e Jones (1993) referem-se como "feedback" negativo. Ao mesmo tempo, outros participantes ficam em uma posição inferiorizada, e estes tendem a tentar "expandir o conflito", ou seja, propiciar a inserção de novos atores nas discussões, fazer com que elas ultrapassem os limites do subsistema e chamem a atenção do grande público. Quando isto ocorre e elementos da macropolítica passam a influenciar naquelas questões de política cria-se uma cadeia de eventos que enseja rápidas mudanças no subsistema.

Um novo ator político que se envolve ou um evento que aloque a atenção das pessoas para aquele subsistema de política, por exemplo, podem deslocar as discussões e a tomada de decisão para novas arenas, nas quais novas imagens de política sejam consideradas e novos atores possam apresentar suas propostas. Assim ocorre o que os autores chamam de "feedback" positivo. Não há um evento específico que cause a mudança, mas sim uma série de acontecimentos mutuamente convergentes. Após ocorrer a mudança a tendência então é que os novos "vencedores" do conflito estabeleçam seu próprio monopólio de política e sigase um novo período de estabilidade. Assim, Baumgartner e Jones (1993) teorizam sua observação empírica, de que as políticas públicas alternam entre longos períodos de incrementalismo com períodos de rápidas e dramáticas mudanças.

Exprime-se a lógica de alternância temporal pela observação de que os mesmos mecanismos que propiciam a estabilidade, dão ensejo, a longo prazo, às pontuações. A consolidação do monopólio de política induz o subsistema à inércia e resulta nas mudanças incrementais nas políticas. Enquanto isso ocorre, os "perdedores" do conflito político estão se organizando, tentando mobilizar a atenção de outros atores e de um público maior para aquelas questões. Ou seja, justamente por estar em uma situação desfavorável, os "perdedores" tendem a buscar novas alternativas, o que em suma entende-se como "expandir o conflito”. Eventualmente, fatores externos, eventos políticos ou sociais e crises acabam por deslocar a atenção de participantes que estavam indiferentes àquelas questões, e essas novas influências tendem a causar as alternâncias nas políticas.

Ao longo dos anos, a "Teoria do Equilíbrio Pontuado" também desenvolveu-se em um modelo de processamento de informações, analisando as pontuações através do estudo da 
Campos Neutrais - Revista Latino-Americana de Relações Internacionais Vol. 1, No 3, p. 56-69, Setembro- Dezembro de 2019

distribuição de atenção e verbas orçamentárias (GREEN-PEDERSEN e PRINCEN, 2016). Em uma ou outra de suas "versões", este modelo foi utilizado nas aplicações empíricas de Rodrigues Neto (2018), Brasil (2017) e Barcelos (2015), cujos trabalhos são analisados a seguir.

\section{Abordagens de Fluxos Múltiplos e Equilíbrio Pontuado em três estudos recentes sobre formação de agenda no Brasil}

Rodrigues Neto (2018), em pesquisa de dissertação, investiga a construção da questão das ações afirmativas em uma universidade pública, utilizando a base teórica de Kingdon (2014) e Baumgartner e Jones (1993). Através de entrevistas e análise documental, conclui que o movimento social negro trabalhou durante muitos anos na construção de uma imagem de política sobre as ações afirmativas, consolidando o entendimento delas como uma reparação às injustiças sofridas pela população negra durante a história do Brasil. Atores individuais atuaram como empreendedores e grupos organizados do movimento estudantil e da sociedade civil pressionaram as autoridades da universidade para que aprovassem políticas de ações afirmativas com um escopo muito maior do que exigia a "Lei de cotas sociais" (Lei 12.711/12). A instituição analisada implementou as cotas em um percentual acima do que exigia e lei, em 2013, e nos anos seguintes aprovou diversas outras políticas de inclusão, como a criação de vagas e processo seletivo específico para indígenas e quilombolas, bolsas de permanência para os alunos cotistas e cotas raciais nos cursos de pós-graduação. Além disso, a pressão exercida pelo movimento estudantil impeliu a universidade a implantar uma política de monitoramento das cotas, no que diz respeito à identificação dos candidatos (RODRIGUES NETO, 2018).

Aplicando especificamente os conceitos de imagem de política, janela de oportunidade e empreendedor de política, e tratando, de forma mais geral, do papel das ideias na construção das políticas, o autor conclui que ideias e visões de mundo dos participantes foram decisivos na formulação das políticas de ações afirmativas da universidade em questão; que o movimento social negro e demais atores proponentes construíram uma imagem consolidada e bem definida, enquadrando as ações afirmativas como um direito de determinados segmentos da população e uma forma de reparar injustiças sofridas por eles ao longo de um processo histórico, cujas consequências se refletem na contemporaneidade; que os proponentes da questão das ações afirmativas aproveitaram-se de uma janela de oportunidade para apresentar novas demandas e "expandir o conflito", promovendo a inserção de grupos ligados à pauta 
dos povos indígenas e outras comunidades que se encontram em situação socioeconômica desfavorável e portanto têm acesso dificultado à universidade pública.

Nem todas essas demandas foram atendidas pela instituição, mas a pesquisa demonstra que as políticas que foram aprovadas têm sua origem nas propostas dos grupos envolvidos e refletem as ideias e imagens construídas por eles e pelos empreendedores.

Brasil (2017), em sua tese de doutorado, utiliza a "Teoria do Equilíbrio Pontuado" para analisar a dinâmica das políticas públicas nos subsistemas de saúde e da assistência social no Brasil. Em suas considerações finais, afirma que "a aplicação da Teoria do Equilíbrio Pontuado lança um novo e promissor caminho sobre os estudos de políticas públicas, em especial aqueles destinados ao acompanhamento e análise da dinâmica das políticas ao longo do tempo" (BRASIL, 2017, p. 244).

Mais especificamente, o autor conclui que, apesar da proximidade temática, políticas de saúde e de assistência social seguem caminhos próprios quanto à captura da atenção governamental. A análise aponta que a saúde é um tema ininterrupto na agenda, com médias de atenção elevadas mesmo nos períodos de estabilidade, enquanto que a assistência social é praticamente ignorada, sendo os momentos não pontuados marcados pela negação do acesso à agenda. Ele identifica dois momentos em que houve grande aproximação nas dinâmicas das duas áreas - na época da promulgação da Constituição Federal de 1988, consolidou-se a imagem da universalidade e da equidade na oferta de serviços de saúde e seguridade; e em meados da década de 1990, houve uma mudança na percepção sobre as políticas sociais em geral, "marcada por cortes de gastos e pela desresponsabilização do Estado (BRASIL, 2017, p. 245).

O autor relaciona os dois momentos distintos com o contexto político mais geral, afirmando que, na época da Constituinte de 1988, em um contexto de redemocratização e consolidação de demandas sociais, abriu-se uma janela de oportunidade para propostas de políticas mais generalistas. Já na década de 1990, em um contexto de crise fiscal e expansão de ideais liberais, firmou-se uma imagem sobre a diminuição da atuação direta do Estado. Se o comportamento das duas áreas foi diferente, a relação dele com o contexto político foi semelhante - em ambos os casos, atores e instituições fomentaram reinterpretações das imagens das políticas estudadas (BRASIL, 2017).

Em sequência, Brasil (2017) aponta conclusões sobre a dinâmica das políticas em cada um dos subsistemas estudados. Afirma que as políticas de saúde permaneceram entre as prioridades dos tomadores de decisão durante todo o período analisado, atribuindo a estabilidade de um considerável nível de atenção à existência de um subsistema coeso, com 
imagens de política bem definidas e bloqueio ao acesso de novos atores. Identifica assim a consolidação de um monopólio de política. Já na área da assistência social, o nível de atenção foi baixo na maior parte do período, com duas pontuações: a primeira, entre 1988 e 1989, quando uma série de legislações é aprovada sob a imagem do papel do Estado como promotor da equidade e da seguridade social; a segunda, entre 1995 e 1998, período em que houve crescimento da atenção sobre as políticas de assistência social, marcado por políticas de combate à pobreza e ações de descentralização e desresponsabilização do Estado, associado ao contexto político de reforma administrativa (BRASIL, 2017).

Por último, falando especificamente sobre a aplicação da "Teoria do Equilíbrio Pontuado" em um estudo de caso no Brasil, o autor comenta sobre a dificuldade de operacionalizar o estudo, pela inexistência de trabalhos anteriores na literatura nacional, e assim, o não conhecimento sobre o modelo ser de fato apropriado para analisar políticas brasileiras, e pela necessidade de construção de um grande banco de dados a da adaptação dos indicadores de atenção utilizados. Enfim, o autor conclui que o modelo do "Equilíbrio Pontuado" é adequado como ferramenta para o estudo das políticas públicas no contexto brasileiro (BRASIL, 2017).

Barcelos (2015), em sua tese de doutorado, estuda o papel das ideias e do conhecimento na construção das políticas públicas da área de biocombustíveis no Brasil. Observa que empreendedores de política, imagem de política e janelas de oportunidade foram conceitos da literatura sobre formação de agenda considerados decisivos na formulação de políticas públicas neste subsistema.

Através da análise da atuação dos empreendedores de política pública como manipuladores de imagens de política, o autor conclui que os outputs de política pública do subsistema foram produzidos a partir das ideias, crenças e visões de mundo dos atores proponentes. Conclui que "foi central o papel dos empreendedores de política pública na formação e mudança na agenda da área de biocombustíveis” (BARCELOS, 2015, p. 221).

O autor identifica políticas relativas aos biocombustíveis etanol e biodiesel. Em ambos os casos, ideias basearam a criação de uma imagem, que permaneceu latente por décadas, até que se abrissem janelas de oportunidades para que as propostas entrassem na agenda governamental. Ao serem implementadas e estabelecerem-se como ideias dominantes naquela comunidade, ensejaram a consolidação de um monopólio de política. Daí seguiu-se longos períodos de estabilidade. As bases para a alternativa do etanol foram construídas durante as décadas de 1920 e 1930, mas o programa referente só seria implementado nos anos 1970. O monopólio que se estabeleceu então, limitaria por mais de 30 anos a ascensão de outras 
alternativas. A opção do biodiesel foi construída de fora para dentro do subsistema, em espaços instituições alheios à "jurisdição" do monopólio vigente, onde novos atores valeramse dessa expansão de conflito e fizeram emergir a ideia de uma política de biocombustíveis com cunho social, ganhando a atenção do presidente da república e da cúpula do poder executivo. $\mathrm{O}$ autor afirma que novas imagens dificilmente iriam emergir se os debates da comunidade permanecessem restritos aos espaços institucionais anteriormente definidos e que a expansão do conflito proporcionou que atores que até então haviam sido "perdedores" na disputa política conseguissem exercer influência na formulação das políticas do subsistema (BARCELOS, 2015).

Metten et al (2015) examinam a ascensão da questão do complexo econômico industrial da saúde (CEIS) na agenda governamental, usando como referencial teórico a Teoria dos Múltiplos Fluxos, de Kingdon (2014). Identificam que a conexão dos fluxos e a atuação de empreendedores de política contribuíram para a tomada de decisão que inseriu o CEIS entre as prioridades do Plano Brasil Maior, do então Ministério da Indústria, Comércio Exterior e Serviços.

Como problema, os autores identificam o déficit da balança comercial do complexo da saúde, a dificuldade de competir internacionalmente e a subsequente necessidade de importar medicamentos. Para desenvolver uma indústria farmacêutica forte e eficiente, atores do subsistema da saúde promoveram o entendimento de que seria necessário adotar um programa governamental para resolver o problema reconhecido. Como solução, Metten et al (2015) identificam a consolidação de uma imagem de política destacando o papel da saúde no desenvolvimento nacional. Importantes atores no campo institucional (Ministério da Saúde, Ministério da Ciência e Tecnologia, BNDES, articulações com a sociedade civil) e acadêmico (grupos de pesquisa, acadêmicos notáveis, congresso da Associação Brasileira de Saúde Coletiva) defenderam essa percepção e consagraram o entendimento da "base produtiva da saúde como indutor de desenvolvimento econômico e social” (METTEN et al, 2015 p. 928). Já no fluxo político, cita-se a aproximação institucional do Ministério da Saúde com as instâncias deliberativas do Plano Brasil Maior, a atuação de empreendedores, o contexto macropolítico favorável e atuação do presidente da república (METTEN et al, 2015).

Os autores identificam empreendedores atuantes em diversos espaços institucionais, promovendo a integração da expertise do complexo da saúde com a política industrial nacional e articulando a priorização do complexo da saúde no projeto de desenvolvimento do país. Destacam também a captura da atenção do presidente da república, expressa na 
Campos Neutrais - Revista Latino-Americana de Relações Internacionais Vol. 1, No 3, p. 56-69, Setembro- Dezembro de 2019

mensagem ao Congresso Nacional de 2013, e o humor nacional favorável no contexto póscrise econômica da primeira década dos anos 2000 (METTEN et al, 2015).

Zapelini (2014) aplica o modelo de Kingdon (2014) à construção de uma política de recursos hídricos em Itajaí, Santa Catarina. Reconhece eventuais limitações do estudo, mas afirma que os principais aspectos da Teoria dos Múltiplos Fluxos são confirmados. O autor analisa a formação de agenda no Comitê Itajaí, responsável por gerir os recursos hídricos da bacia hidrográfica do Rio Itajaí. Enquadra os usuários como empreendedores e observa que estes aproveitaram-se de uma janela de oportunidade e influenciaram a definição da cobrança pelo uso da água. Afirma que:

os empreendedores de política do setor dos usuários aproveitaram a janela de oportunidade e levaram o Comitê a abandonar sua formulação original para a cobrança e aceitar sua modificação, constituída com a participação efetiva de representantes desses empreendedores. (...) uma vez que as oportunidades surgem, grupos bem organizados tiram proveito delas para rediscutir ou modificar questões e aspectos que aparentemente já eram consensuais (ZAPELINI, 2014, p. 809-810).

Primeiramente, pode-se afirmar que os modelos teóricos de Kingdon (2014) e de Baumgartner e Jones (1993) são claros o bastante para serem aplicados empiricamente, sem que haja confusão teórica ou que eventuais ambiguidades comprometam a validade dos estudos. Principalmente o modelo de Kingdon (2014) já foi criticado por ser essencialmente aleatório e não produzir conhecimento científico, porém revisões como as de Jones e Cairney (2016), Capella (2006), e a adição do próprio Kingdon à versão original de sua obra “Agendas, Alternatives and Public Policies" destacam que a aleatoriedade é residual em seu modelo e que as variáveis de cada fluxo permitem a observação empírica com satisfatório grau de confiabilidade. De acordo com estes autores, a grande contribuição da "Teoria dos Múltiplos Fluxos" é exatamente a sua maleabilidade para tratar do complexo sistema político.

Quanto à sua aplicabilidade no contexto brasileiro, os estudos revisados neste artigo revelam, em grande parte, que os conceitos desenvolvidos por Kingdon (2014) podem ser utilizados para analisar a formulação de políticas públicas no Brasil. Em todos os casos que utilizam a "Teoria dos Múltiplos Fluxos", seus autores concluíram que empreendedores de política e janelas de oportunidade foram decisivos nos processos analisados. Rodrigues Neto (2018) e Barcelos (2015) destacam especialmente o papel das ideias e sua transformação em alternativas legítimas de políticas. Outro ponto de congruência é a corroboração da ideia de 
“amaciamento" ("softening up"), de Kingdon (2014), referente à necessidade de "preparar o terreno" e persistir durante muito tempo, até que uma proposta de política seja de fato implementada. Nos dois estudos citados, as "soluções" foram desenvolvidas durante décadas, até que finalmente o contexto político proporcionasse a abertura de uma "janela de oportunidade”. A pesquisa de Barcelos (2015) também corrobora a importância do presidente da república, cuja atenção foi considerada fundamental para que as políticas de biocombustíveis fossem consideradas no contexto macropolítico.

De Baumgartner e Jones (1993), destaca-se a noção de imagem de política, expressa na pesquisa de Rodrigues Neto (2018) pela narrativa que enquadra as ações afirmativas como reparações históricas e em sua aplicação em detrimento a outras imagens, que destacavam o impacto que essas políticas teriam na instituição em questão e a ineficiência de aplicá-las no ensino superior, entre outros enquadramentos. Os estudos de Brasil (2017), Barcelos (2015) e Metten et al (2015), em comum, apontam que a imagem das políticas de seus subsistemas como instrumentos de desenvolvimento social foi fundamental para engajar novos atores e conquistar a atenção das autoridades, moldando as ações que viriam a ser implementadas. Em todos os casos, a influência do contexto político mais geral sobre a formulação de políticas públicas foi considerada decisiva. Brasil (2017) relaciona o ambiente da redemocratização à consagração das imagens das políticas de saúde e assistência social de cunho universal. Barcelos (2015) e Metten et al (2015) destacam o "humor nacional" favorável à políticas de desenvolvimento, em um contexto de crescimento econômico e inserção do Brasil como ator importante da política internacional.

Do estudo de Zapelini (2014), muito mais conciso, destaca-se a importância dos empreendedores de política e do aproveitamento de uma janela de oportunidade. Em geral, a ideia de Kingdon (2014) sobre a imprevisibilidade da janela e a relação da formulação de políticas públicas com o modelo da "lata de lixo" (COHEN, MARCH e OLSEN, 1972) é corroborada pelos resultados de Rodrigues Neto (2018) e Zapelini (2014), os quais observam que, quando as questões analisadas conquistaram a atenção dos tomadores de decisão, os participantes aproveitaram-se para apresentar novas demandas.

Enfim, conforme afirmado na introdução, esta revisão não visa apresentar resultados absolutos, mas sim colaborar com a produção brasileira sobre formação de agenda e subsidiar outras pesquisas que apliquem os modelos citados em estudos de caso no Brasil. 
Campos Neutrais - Revista Latino-Americana de Relações Internacionais Vol. 1, No 3, p. 56-69, Setembro- Dezembro de 2019

\section{Considerações finais}

Este artigo teórico teve como objetivo promover uma aproximação da literatura sobre formação de agenda com o ambiente da formulação de políticas no Brasil. Como dizem alguns dos autores revisados, a carência de dados e de corroboração sobre a aplicabilidade dos modelos mais consagrados ao contexto brasileiro dificulta a realização de novos estudos. Assim, espera-se, com esta revisão, apresentar de forma ágil os resultados de algumas das mais recentes investigações sobre formação de agenda no Brasil.

Concluiu-se que as principais proposições de Kingdon (2014) e Baumgartner e Jones (1993) são aplicáveis ao contexto brasileiro, principalmente os conceitos de imagem de política, empreendedor de política e janela de oportunidade. As noções gerais de Kingdon sobre a transformação de ideias em alternativas de ação material pelo governo e instituições públicas são confirmadas, afinal, como se presume na literatura sobre formação de agenda, as políticas públicas não são geradas de forma espontânea, mas sim construídas ao longo do tempo pelas pessoas que atuam nas comunidades ou subsistemas de política.

Eventuais limitações deste artigo são a quantidade limitada de dados analisados e a possibilidade de que outros modelos teóricos possam ser aplicados para os estudos em questão. Novas revisões e pesquisas poderão trazer grandes contribuições, compilando mais dados ou utilizando outros modelos teóricos que analisem a formulação de políticas públicas, como a "Advocacy Coallition Framework" ou a "Narrative Policy Framework".

\section{Bibliografia}

BARCELOS, Márcio. Ideias, agendas e políticas públicas: Um estudo sobre a área de biocombustíveis no Brasil. Tese (Doutorado em Sociologia), Universidade Federal do Rio Grande do Sul - UFRGS, Porto Alegre, 2015.

BAUMGARTNER, Frank; JONES, Bryan. Agendas and instability in American politics. University of Chicago Press. Chicago, 1993.

BRASIL, Felipe Gonçalves. A dinâmica das políticas de saúde e assistência social no Brasil: Incrementalismo e pontuações na atenção governamental entre 1986 e 2003. Tese (Doutorado em Ciência Política), Universidade Federal de São Carlos - UFSCar, São Carlos, 2017.

CAPELlA, Ana Cláudia. Perspectivas Teóricas sobre o Processo de Formulação de Políticas Públicas. Revista Brasileira de Informação Bibliográfica em Ciências Sociais, São Paulo, n. 61, p. 25-52, 1º semestre, 2006.

COHEN, Michael D.; MARCH, James G.; OLSEN, Johan P.. A garbage can model of organizational choice. Administrative Science Quarterly, Ithaca, NY, v. 17, n. 1, p.1-25, mar. 1972. 
Campos Neutrais - Revista Latino-Americana de Relações Internacionais Vol. 1, No 3, p. 56-69, Setembro- Dezembro de 2019

GREEN-PEDERSEN, Christoffer; PRINCEN, Sebastiaan. Punctuated Equilibrium Theory. In: ZAHARIADIS, Nikolaos (Ed.). Handbook of public policy agenda setting. Edward Elgar Publishing, 2016. p. 69-86.

JONES, Michael D.; CAIRNEY, Paul. Kingdon's Multiple Streams Approach: What Is the Empirical Impact of this Universal Theory?.Policy Studies Journal, v. 44, n. 1, p.37-58, fev. 2016. Disponível em: https://onlinelibrary.wiley.com/doi/10.1111/psj.12111. Acesso em: 22 mar. 2019.

KINGDON, John. Agendas, alternatives and public policies. Pearson New International Edition. Pearson Educated Limited, 2014.

METTEN, Antoine et al. A introdução do complexo econômico industrial da saúde na agenda de desenvolvimento: uma análise a partir do modelo de fluxos múltiplos de Kingdon. Revista de Administração Pública, Rio de Janeiro, v. 49, n. 4, p. 915-936, jul./ago. 2015. Disponível em: http://bibliotecadigital.fgv.br/ojs/index.php/rap/article/view/51568/50353. Acesso em: 23 mar. 2019.

ROCHEFORT, David A.. Agenda setting, problem definition, and their contributions to a political policy analysis. In: ZAHARIADIS, Nikolaos (Ed.). Handbook of public policy agenda setting. Edward Elgar Publishing, 2016. p. 35-52.

RODRIGUES NETO, Damasio Duval. Formação de agenda em uma universidade pública: A construção da questão das ações afirmativas. Dissertação (Mestrado em Administração Pública), Universidade Federal de Pelotas - UFPel, Pelotas, 2018. Disponível em: https://wp.ufpel.edu.br/profiap/files/2018/12/TCF-Damasio.pdf. Acesso em: 25 mar. 2019.

ZAHARIADIS, Nikolaos. Handbook of public policy agenda setting. Edward Elgar Publishing, 2016. 505 p.

ZAPELINI, Marcello Beckert. Montagem de agenda no Comitê Itajaí: Uma aplicação do modelo de Kingdon. Revista de Administração Contemporânea, Curitiba, v. 18, n. 6, p. 795-812, nov./dez. 2014. Disponível em: <https://rac.anpad.org.br/index.php/rac/article/view/1071/1067〉. Acesso em: 23 mar. 2019. 